\title{
End Stage Palliative Care of Head and Neck Cancer: a Case Study
}

\author{
Nitin Pratap Shishodia ${ }^{1}$, Darshan Devang Divakar ${ }^{2 *}$, Abdulaziz Abdullah Al \\ Kheraif ${ }^{2}$, Ravikumar Ramakrishnaiah ${ }^{2}$, Akbar Ali Khan Pathan ${ }^{3}$, Narasimha \\ Reddy Parine ${ }^{3}$, Santhosh Vediyera Chandroth ${ }^{4}$, Binu Purushothaman ${ }^{4}$
}

\begin{abstract}
Background: Locally advanced head and neck cancer is generally incurable and has a short survival rate. This study aimed to evaluate symptom relief, disease response, and acute toxicity after palliative hypo-fractionated radiotherapy and long-term survival in affected patients. Materials and Methods: Between January 2011 to December 2011, 80 patients who were histopathologically diagnosed as having stage III or stage IV head and neck squamous cell carcinoma based on Eastern Cooperative Oncology Group (ECOG) performance status 1-3, were offered palliative radiotherapy $(20 \mathrm{~Gy} / 5 \mathrm{Fr} / 5$ Days). Later these patients were evaluated on 30th day after completion of treatment for disease response based on World Health Organisation (WHO) criteria and palliation of symptoms using symptomatic response grading and acute toxicities by the Radiation Therapy Oncology Group (RTOG). Many patients were given post radiation therapy (RT) palliative chemotherapy for appropriate palliative care and a few patients were selected for further curative RT. The overall survival was also evaluated among this group of patients with last follow up date of 1st May, 2014. Results: The most common presenting complaint was pain followed by dysphagia. Most patients $(60-70 \%)$ had appreciable relief in their presenting symptoms. A good response was observed in the majority following palliative RT; a few patients had progressive disease and some had stable and regressed disease. None of the patients experienced radiation toxicity that required hospital admission. Almost all showed grade one and two acute skin and mucosal toxicity one month after completion of treatment. The mean survival days for patients given only hypofractionated palliative RT was 307 days, those with post palliative RT and palliative chemotherapy was 390 days and patients who went on to receive further palliative RT and curative RT dose had significantly overall survival of $\mathbf{5 8 2}$ days. Conclusions: Advanced head and neck cancer should be identified for suitable palliative hypofractionated radiotherapy to achieve acceptable symptom relief in a great proportion of patients and should be followed by palliative chemotherapy or curative RT in suitable cases for long-term symptom-free survival.
\end{abstract}

Keywords: Cancer of head and neck - palliative therapy - radiation therapy - survival rate

Asian Pac J Cancer Prev, 16 (3), 1255-1258

\section{Introduction}

Head and neck cancer is one of the predominant cancers in developing countries like India (Takiar and Vijay, 2011; Das et al., 2013). Population-based cancer registry in India reported that the number of tobaccorelated cancer and head and neck cancer would be around 3,16,734 and 2,18,421 respectively, by 2020 (Jayaraj et al., 2014). In utmost cases, due to widespread locoregional involvement, poor condition of the patient or comorbid circumstances curative treatment is not possible. Therefore, the significance of aggressive treatment in unresectable locally advanced head and neck cancer has been examined. The intent of treatment in such cases is to enhance the quality of life of the patients keeping their socioeconomic condition in mind and cautiously utilizing the precious resources for curable conditions (Das et al., 2013).

The recent cancer estimates show that out of 14.1 million new cancer cases diagnosed annually more than 0.7 million patients suffer from head and neck cancer and $60 \%$ of all head and neck cancer occur in developing countries. The 5-year survival rates of multimodal chemoradiotherapy are below $20 \%$, with a median survival of 12 months or less (Mishra and Meherotra, 2014). As per World Health Organization 'Palliative Care' is an approach that

${ }^{I}$ Department of Oral Medicine and Radiology, Healthy Smiles Dental Clinic, New Delhi, ${ }^{4}$ Department of Periodontics and Orthodontics, KMCT Dental College, Kerala University of Health and Allied Science, Kerala, India, ${ }^{2}$ Dental Biomaterials Research Chair, College of Applied Medical Sciences, ${ }^{3}$ Genome Research Chair, Department of Biochemistry, College of Science, King Saud University, Riyadh, Kingdom of Saudi Arabia*For correspondence: darshandevang@gmail.com 
enhances the quality of life of patients and their families facing the difficulties associated with life-threatening illness, through the prevention and relief of suffering by means of timely identification, impeccable assessment, treatment of pain and other physical, psychosocial and spiritual problems (Sharifa Ezat et al., 2014). Stress should be placed on educating phsyicians about palliative care (Budkaew and Chumworathayi, 2013).

It is apparent that palliative treatment may vary from curative treatment but in certain situations the borderline between them may be blurred and a clear-cut distinction may not always be possible in many $\mathrm{T} 3$ and $\mathrm{T} 4$ primary lesions or recurrent diseases (Mitra et al., 2006). Initially in the course of illness, it is necessary to combine chemotherapy and radio therapy with other therapies in order to prolong the life and include those investigations necessary to better understand and manage distressing clinical complications.

The present study is intended to evaluate the response and radiation toxicity of short course hypofractionated radiation for locally advanced head and neck cancer and to assess the development of signs and symptoms on the basis of clinical observation and subjective feeling followed by observation of long-term survival in these patients.

\section{Materials and Methods}

In our study, 80 advanced head and neck cancer patients were included from Jan 2011 to December 2011. Patients with stage III \& IV AJCC (American Joint Committee on Cancer), European Cooperative Oncology Group (ECOG) performance status $(1,2,3)$ with life expectancy <1year were included. Patients were treated by external beam radiotherapy delivered by Cobalt- 60 teletherapy machine (Theratron $780 \mathrm{E} / 780 \mathrm{C}$ ). A total dose of 20Gy was given in 5 fractions in 5 consecutive days with a dose of $4 \mathrm{~Gy}$ per fraction. Treatment volume included primary tumor site plus neck region in cases with nodal metastasis. Bilateral parallel opposed fields were planned where disease crossing the midline and had bilateral presentation and dose was being prescribed to midline. These patients were evaluated on 30th day and assessed for treatment response in terms of disease control using WHO criteria and palliation of symptoms using symptomatic response grading (Paliwal et al., 2012). Acute skin and mucosal reactions grading was done as per RTOG (Radiation Therapy Oncology Group) toxicity criteria. Further treatment of patients was done according to tumor regression status; most of these patients were selected for palliative chemotherapy for further palliation and few for curative RT dose of 70Gy equivalent. Then, overall survival in these groups of patients was evaluated.

\section{Results}

Characteristics of the patient, disease and treatment are shown in (Table 1). Mean age was 66 years ranging from 37-90 years. Most of patients had ECOG performance status 1 and 2 with common presenting complaint as pain followed by dysphagia. Buccal mucosa and tongue were commonly involved primary sites proven histopathologically as moderately differentiated squamous cell carcinoma.

Treatment related toxicities such as acute skin reaction was $69-86 \%$ in grade I and $11-14 \%$ in grade II involvement patients, acute mucosites was $65-81 \%$ in grade I, $11-14 \%$ in grade II and 4-5\% in grade III involvement patients. None of the patients experienced radiation toxicities that required hospitalisation. Almost all patients showed grade one and two acute skin and mucosal toxicities at one month after completion of treatment.

Symptom burden at presentation and symptomatic relief on follow-up visits are shown in (Table 2). Majority of patients $(60-80 \%)$ had appreciable relief in their presenting symptoms. On overall treatment response after one month follow-up $60 \%$ patients showed good response (GR), 19\% patients showed partial response, $14 \%$ patients showed poor response and $7.5 \%$ patients showed no response respectively. In a long-term follow up till 1st may, 2014 it was observed that patients who were given only palliative RT had a mean survival time

Table 1. Characteristics of the Patient, Disease and Treatment

\begin{tabular}{clc}
\hline Variable & & No. of patients \\
\hline SEX & Male & $60(75 \%)$ \\
& Female & $20(25 \%)$ \\
ECOG & I & $53(66 \%)$ \\
& II & $20(25 \%)$ \\
III & $7(9 \%)$ \\
MAIN COMPLAINT & \\
Pain & $75(94 \%)$ \\
Dysphagia & $12(15 \%)$ \\
& Hoarseness & $11(14 \%)$ \\
Respiratory distress & $7(9 \%)$ \\
Others & $4(5 \%)$ \\
PRIMARY SITE OF DISEASE & \\
Buccal mucosa & $20(25 \%)$ \\
Tongue & $14(17.5 \%)$ \\
Hard palate and alveolus & $6(7.5 \%)$ \\
Unknown primary & $6(7.5 \%)$ \\
Oropharynx & $12(15 \%)$ \\
& Hypo pharynx & $9(11 \%)$ \\
Larynx & $6(7.5 \%)$ \\
Others & $7(9 \%)$ \\
STAGE & III & $18(22.5 \%)$ \\
& IV & $62(77.5 \%)$ \\
\hline
\end{tabular}

Table 2. Symptom Burden at Presentation and Symptomatic Relief on Follow-Up Visits

\begin{tabular}{|c|c|c|c|}
\hline \multirow[t]{2}{*}{ Main symptom on presentation (no. of patients) } & \multicolumn{3}{|c|}{ Symptom Relief } \\
\hline & No relief $(\%)$ & Partial relief $(\%)$ & Appreciable relief $(\%)$ \\
\hline Pain & $12(16 \%)$ & $15(20 \%)$ & $48(64 \%)$ \\
\hline Dysphagia & 0 & $4(33 \%)$ & $8(67 \%)$ \\
\hline Hoarseness of voice & $2(18 \%)$ & $2(18 \%)$ & $7(64 \%)$ \\
\hline Respiratory distress & $1(14 \%)$ & $2(28 \%)$ & $4(57 \%)$ \\
\hline
\end{tabular}


of 307 days and those who received palliative RT and palliative chemotherapy had mean survival time of 390 days and those patients who went on to receive palliative RT with further curative RT dose had significantly overall survival time of 582 days. Among all, $6.25 \%$ of patients were known to be alive.

\section{Discussion}

Although it is appropriate to have a well-coordinated multimodal approach for advanced head and neck cancer, there are many grey areas in treatment since both stage III and stage IV patients and resectable/unresectable patients were combined in the reported studies. In a study by Vikram (2003) reports that advanced head and neck cancers from developing regions do not show favourable results and it would seem prudent for these patients to explore innovative ways of arranging relief. It is now identified that a part of advanced stage patients have life-limiting disease. Weissberg et al. (1983) assessed conventionally fractionated versus hypofractionated palliative External Beam Radiation Therapy (EBRT) schedules for patients with locally recurrent or advanced head and neck cancers. They have compared 60 Gy to $70 \mathrm{~Gy}$ in six to seven weeks against $40 \mathrm{~Gy}$ to $48 \mathrm{~Gy}$ in 64 patients with stages III and IV surgically unresectable squamous cell carcinoma. No variances were observed in tumor control, acute side effects, or long term sequelae.

In another study by Ghoshal et al. (2004), they have evaluated the role of palliative radiotherapy for symptom control in patients with locally advanced head and neck cancer. In this experiment, 25 patients with stage 3 and 4 head and neck cancer were treated with a short course of palliative radiotherapy 30 Gray (Gy) in 10 fractions over 2 weeks. Initial symptoms were measured using an 11 point numerical scale for pain, dysphagia, cough, insomnia and dyspnoea. The prime end point was relief of symptoms in the 4th week after radiotherapy. Every patient with pain and $90 \%$ of patients with dysphagia, dyspnoea and disturbed sleep had more than $50 \%$ relief in symptoms after radiotherapy.

In a similar study by Paliwal et al. (2012) reported that $52 \%$ patients presented with pain and $32 \%$ of patients with dysphagia, after radiotherapy more than $76 \%$ got relief from pain and more than $66 \%$ patients got relief from dysphagia. Mohanti et al. (2004) examined 505 patients with stage IV head and neck squamous cell carcinoma, and they observed a uniform regimen of $20 \mathrm{~Gy} / 5$ fractions, once daily over 1 week. They reported good symptomatic relief (>50\% for pain, $53 \%$ for dysphagia, $57 \%$ hoarseness, $47 \%$ otalgia, $76 \%$ for respiratory distress and $59 \%$ for cough). At one month assessment, $37 \%$ achieved a partial response and had ambulatory physical state suited for further curative-dose radiotherapy. Ling et al. (1996) assessed the effect of Electron Beam Intraoperative Radiotherapy (EB-IORT) on local-regional control and any associated problems in patients with head and neck cancer. In this study, EB-IORT was given as a single fraction of $1500 \mathrm{cGy}$ to the $90 \%$ isodose with 6 or $9 \mathrm{Mev}$ electrons. After 30 months of follow-up, $27 \%$ patients had only local recurrence. Of these, only one recurrence was inside the EB-IORT field and eight were outside the EB-IORT field. Seven percent patients developed distant metastases only and three percent patients had both local recurrence and distant metastasis. In patients who are known to be alive, $68 \%$ of them have no evidence of disease. The 3-year actuarial local-regional control rate was $60 \%$. They concluded that due to unavailability of IORT in developing countries this modality remains underused.

Our study which was also conducted in similar way, reported symptomatic relief (more than $80 \%$ for pain, $70 \%$ for dysphagia, $60 \%$ for hoarseness, and $70 \%$ for respiratory distress). The main acute toxicity of palliative radiotherapy was similar to above study. After one month of assessment $79 \%$ patients achieved appreciable response. Our data supports the findings of Paliwal et al. (2012). Fraction size is the dominant factor in determining late effects but overall treatment time has little influence on these effects. In patients having poor survival, late effects are meaningless; it is a determining factor in providing good quality of life. Thus, patients in whom disease progressed in spite of hypofractionated radiotherapy were offered other palliative symptomatic treatment. We conclude that adverse advanced stage head and neck cancer can be identified for a suitable short course palliative radiotherapy which will achieve growth restraint and symptom relief in sizeable proportions of patients. This study tries to strike a balance between economic burden, treatment time and hospital stay and machine load. Advanced head and neck cancer should be identified for suitable palliative hypofractionated radiotherapy to achieve acceptable symptom relief in great proportion of patients and should be followed by palliative chemotherapy or curative RT in suitable cases for long-term symptom-free survival.

\section{Acknowledgements}

The authors would like to express their appreciation to the Research Centre, College of Applied Medical Sciences and the Deanship of Scientific Research at King Saud University, Riyadh, Kingdom of Saudi Arabia for funding this research.

\section{References}

Budkaew J, Chumworathayi B (2013). Knowledge and attitudes toward palliative terminal cancer care among Thai generalists. Asian Pac J Cancer Prev, 14, 6173-80.

Das S, Thomas S, Pal SK, et al (2013). Hypofractionated palliative radiotherapy in locally advanced inoperable head and neck cancer: CMC vellore experience. Indian J Palliat Care, 19, 93.

Ghoshal S, Patel F, Mudgil N, et al (2004). Palliative radiotherapy in advanced head and neck cancer-A prospective trial. Indian J Palliat Care, 10, 19-23.

Jayaraj R, Singh J, Baxi S, et al (2014). Trends in incidence of head and neck cancer in the Northern Territory, Australia, between 2007 and 2010. Asian Pac J Cancer Prev, 15, 7753.

Ling SM, Roach M, Fu KK, et al (1996). Local control after the use of adjuvant electron beam intraoperative radiotherapy in patients with high-risk head and neck cancer: the UCSF 
Nitin Pratap Shishodia et al experience. Cancer J Sci Am, 2, 321-9.

Mishra A, Meherotra R (2014). Head and neck cancer: global burden and regional trends in India. Asian Pac J Cancer Prev, 15, 537-550.

Mitra J, Mishra S, Bhatnagar S (2006). Advanced head \& neck cancer: care beyond cure. Internet J Pain Symptom Control Palliat Care, 4, 2.

Mohanti BK, Umapathy H, Bahadur S, et al (2004). Short course palliative radiotherapy of $20 \mathrm{~Gy}$ in 5 fractions for advanced and incurable head and neck cancer: AIIMS study. Radiother Oncol, 71, 275-80.

Paliwal R, Patidar AK, Walke R, et al (2012). Palliative hypofractionated radiotherapy in locally advanced head and neck cancer with fixed neck nodes. Iranian J Cancer Prev, 5, 178-82.

Sharifa Ezat WP, Fuad I, Hayati Y, Zafar A, Wanda Kiyah GA (2014). Observational study on patient's satisfactions and quality of life (QoL) among cancer patients receiving treatment with palliative care intent in a tertiary hospital in Malaysia. Asian Pac J Cancer Prev, 15, 695-701.

Vikram B (2003). Cancers of the head and neck region in developing countries. Radiother Oncol, 67, 1-2.

Weissberg JB, Pillsbury H, Sasaki CT (1983). High fractional dose irradiation of advanced head and neck cancer. Implications for combined radiotherapy and surgery. Arch Otolaryngol, 109, 98-102. 